\title{
Reestruturando a Área de Tecnologia da Informação: um relato de experiência na administração pública
}

\author{
Alternative Title: Restructuring Information Technology Area: an experience \\ report in the public service
}

\author{
Fernando Szimanski \\ Ministério da Ciência, \\ Tecnologia e Inovação - MCTI \\ Esplan. dos Ministérios, BI. E \\ Brasília - DF, Brasil 70067-900 \\ fernando.szimanski@ \\ mcti.gov.br \\ George H. Kuroki Jr. \\ Ministério da Ciência, \\ Tecnologia e Inovação - MCTI \\ Esplan. dos Ministérios, BI. E \\ Brasília - DF, Brasil 70067-900 \\ george.junior@mcti.gov.br
}

\author{
Anivaldo S. Vale ${ }^{\dagger}$ \\ Ministério da Ciência, \\ Tecnologia e Inovação - MCTI \\ Esplan. dos Ministérios, BI. E \\ Brasília - DF, Brasil 70067-900 \\ anivaldo.vale@mcti.gov.br \\ Célia G. Ralha \\ Universidade de Brasília - UnB \\ Campus Universitário Darcy \\ Ribeiro - Asa Norte - CIC/EST \\ Brasília - DF, Brasil 70910-900 \\ ghedini@cic.unb.br
}

\begin{abstract}
RESUMO
Um dos maiores desafios da área de Tecnologia da Informação (TI) de uma organização é oferecer suporte eficaz e eficiente para a tomada de decisão das áreas finalísticas no alcance de sua missão, metas, indicadores e atendimento às exigências legais. A chave para vencer este desafio está na geração de Sistemas de Informação, a partir de modelos de negócio que, bem analisados e adequadamente especificados, originam produtos que usem como matéria prima a realidade do negócio e os objetivos estratégicos. Este trabalho apresenta a experiência adquirida com uma proposta de reestruturação global, a qual inclui desde a reformulação de processos internos até a customização e implantação de ferramentas de suporte ao desenvolvimento de software, implicando em um movimento no sentido de uma mudança da
\end{abstract}

\footnotetext{
*Coordenador do Escritório de Projetos de Tecnologia da Informação - EPTI/MCTI

†Coordenador-substituto do Escritório de Projetos de Tecnologia da Informação - EPTI/MCTI

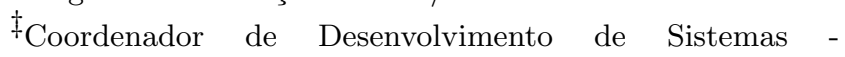
CODS/MCTI

$\S_{\text {Consultora em Melhoria de Processos e Sistemas de Infor- }}$ mação - CIC/UnB
}

Permission to make digital or hard copies of all or part of this work for personal or classroom use is granted without fee provided that copies are not made or distributed for profit or commercial advantage and that copies bear this notice and the full citation on the first page. To copy otherwise, to republish, to post on servers or to redistribute to lists, requires prior specific permission and/or a fee.

SBSI 2015, May 26th-29th, 2015, Goiânia, Goiás, Brazil

Copyright SBC 2015. cultura organizacional. A proposta foi implantada através de uso de um arcabouço em diversos projetos internos de TI no Ministério da Ciência, Tecnologia e Inovação. Ao completar o primeiro ciclo de execução foram verificados alguns benefícios organizacionais, tais como o aumento na qualidade dos serviços de TI, a satisfação e o envolvimento do cliente, bem como a transparência na condução dos projetos de TI.

\section{Palavras-Chave}

Tecnologia da Informação, Sistemas de Informação, Melhoria de Processos, Administração Pública Federal

\begin{abstract}
One of the biggest challenges for an organizations IT area is to provide support so effective and efficient that the finalistics areas can achieve their missions, goals, indicators and compliance with legal requirements. The key to this problem is associated to information systems which are based on business models, being properly analyzed and specified, originating products that are close to business objectives and strategies. This article presents the experience with a complete restructuring proposal of the IT area, which includes changing internal processes, customization and deployment of software tools, involving organizational cultural changes. The proposal was implemented with the help of a framework, being used in many IT projects at the Ministry of Science, Technology and Innovation. When Complete the first execution cycle some organization benefits were verified, such as IT services quality, customer satisfaction and engagement, as well as transparency on IT projects.
\end{abstract}




\section{Categorias e Descritores do Assunto}

D.2.0 [Engenharia de Software]: Geral; H.1.m [Sistemas de Informação]: Miscelânea

\section{Termos Gerais}

Gerenciamento, Padronização, Aspectos Legais

\section{Keywords}

Information Technology, Information Systems, Process Improvement, Public Service

\section{INTRODUÇÃO}

O estabelecimento de um mecanismo corporativo para garantir o alinhamento e alavancagem das decisões de gerenciamento de Tecnologia da Informação (TI) com as estratégias de negócio e operacionais, planos e prioridades apresenta um desafio importante para as organizações atuais. Experiências em diversas organizações públicas e privadas ressaltam o fato de que os Sistemas de Informação (SI) são sistemas sociais complexos. Desta forma, a coordenação da estratégia de TI dentro do contexto de grandes departamentos governamentais requer uma abordagem holística, a fim de realizar ambas as transformações culturais e estruturais, necessárias para superar a inércia política e burocrática dos mecanismos tradicionais verticais de controle e responsabilidade [3].

Diante de tal situação, há de se considerar que trata-se de um grande desafio para a área de TI, a qual precisa manipular novas tecnologias de forma acelerada, mas depende de processos não tão ágeis de governança e gestão relacionados às atividades de desenvolvimento de SI. Assim como as organizações privadas, as públicas demandam respostas rápidas aos requisitantes de serviços de TI, principalmente no que tange ao desenvolvimento de SI eficazes e eficientes, que viabilizem a melhoria na qualidade do trabalho, utilizando de forma racional os recursos escassos, reduzindo custos, identificando gargalos nos processos, enfim, agregando valor ao negócio organizacional.

Na literatura diversos trabalhos foram propostos para endereçar problemas no gerenciamento de projetos de TI, bem como na manipulação de tecnologias relacionadas ao desenvolvimento e manutenção de SI. Por exemplo, da Silva et al. [2] apresentam conceitos relacionados ao gerenciamento de projetos de TI em um projeto de inovação, apresentando um estudo de caso com o objetivo de demonstrar as potencialidades e oportunidades da abordagem proposta. Na Administração Pública Federal (APF), observa-se diversas iniciativas de desenvolvimento de SI, dentre elas destacase o trabalho proposto por Lima et al. [9], relacionado ao desenvolvimento de um SI que auxilia na transição governamental. Embora não sejam propostas voltadas para uma completa reestruturação da área de TI, apresentam aspectos da complexa gestão de projetos de TI no âmbito da APF.

Nesse contexto, o objetivo deste trabalho é apresentar um relato de experiência no âmbito da Coordenação de Desenvolvimento de Sistemas (CODS) do Ministério da Ciência, Tecnologia e Inovação (MCTI), órgão integrante da APF. Este relato descreve o projeto de melhoria da área de TI, o qual utiliza um arcabouço de reformulação e implantação de processos, ferramentas, técnicas, cultura organizacional, entre outras características que em conjunto são capazes de fomentar o desenvolvimento de SI através do aumento da maturidade organizacional.

Considerando um relato de experiência na APF, destacase como principais contribuições do trabalho: proposta de um conjunto de processos internos, o qual pode ser utilizado como guia para o desenvolvimento e manutenção de projetos de TI em órgãos da APF; experiência de implantação de um Escritório de Projetos na APF, consoante com a proposta de um Project Management Office (PMO) [10]; alinhamento de processos internos com a legislação vigente para contratação e gestão de serviços de TI no âmbito público, considerando os diversos órgãos de controle da União e suas respectivas exigências; detalhamento da estratégia adotada com fins a mudança da cultura organizacional; relato dos resultados preliminares alcançados com a reestruturação da área de TI em um órgão da APF.

O restante do artigo está estruturado da seguinte forma: na Seção 2 é apresentada uma pequena parcela dos fundamentos do trabalho contextualizando no âmbito do MCTI; na Seção 3 é discutido o projeto de reestrutuação, em particular apresenta a base de técnica utilizada como estratégia da restruturação de TI; na Seção 4 são apresentados os resultados preliminares da implantação do projeto; e finalmente, na Seção 5 o artigo é concluído.

\section{FUNDAMENTAÇÃO TEÓRICA}

Visando construir o conhecimento acerca dos principais conceitos envolvidos, esta seção apresenta uma revisão de conceitos relacionados ao gerenciamento de SI, bem como um histórico e situação atual da prestação de serviços de TI na $\mathrm{APF}$.

\subsection{Gerenciamento de SI}

Os SI são parte indispensável nas organizações, podendo afetar, das mais variadas formas, o desempenho organizacional. O uso estratégico de SI é capaz de prover novas oportunidades de negócios, para que se possa atingir vantagens competitivas. Um SI pode ser definido como um conjunto de componentes inter-relacionados que coletam, processam, armazenam e distribuem informações para apoiar à tomada de decisão, coordenar e controlar uma organização. Além disso, os SI também podem ajudar os gestores e colaboradores na análise de problemas, visualização de assuntos complexos e criação novos produtos [8].

Os gestores ao perceberem os desafios de negócios, definem a estratégia da organização atuando na alocação dos recursos humanos e financeiros, bem como na coordenação do trabalho. O trabalho de gestão é enfrentar a situações adversas e formular planos de ação para resolver os problemas organizacionais.

\subsection{Prestação de serviços de TI na APF}

Os SI voltados ao negócio proporcionam uma ferramenta de apoio aos gestores atuais, e como a APF tem como um de seus princípios fundamentais a eficiência (EC 19/98), devese buscar um aperfeiçoamento na prestação dos serviços públicos, mantendo ou melhorando os serviços com economia de despesas. Tendo sempre como objetivo a qualidade nos serviços e racionalidade nos gastos.

\subsubsection{Histórico}

Nas últimas décadas, através de pesquisas sobre governança de TI nos órgãos e entidades federais, o Tribunal de 
Contas da União (TCU) constatou que não havia Governança de TI nos órgãos públicos, os contratos não estavam sendo gerenciados de forma correta e nem fiscalizados de maneira eficiente. Não existiam, nos contratos de TI, indicadores de qualidade, níveis de serviços, parâmetros de performance e cláusulas de multas ou penalidades que inibissem a má prestação de serviços por parte das empresas terceirizadas.

Essas pesquisas do TCU resultaram em diversas recomendações, tais como: (1) MPOG/SLTI -- criação da Instrução Normativa MP/SLTI No 4/2010 ${ }^{1}$, denominada "IN 04", que fornece um guia prático para a contratação de soluções de TI; (2) MPOG/SLTI - fortalecimento do Sistema de Administração dos Recursos de Tecnologia da Informação (SISP). Órgão instituído com o objetivo de organizar a operação, controlar, supervisionar e coordenar os recursos de informação e informática da administração direta, autárquica e fundacional do Poder Executivo Federal; (3) MPOG/SLTI - execução de concurso público para Analista de TI, tendo como principais atribuições atividades de planejamento, supervisão, coordenação e controle dos recursos de TI relativos ao funcionamento da APF; (4) Escola Nacional de Administração Pública (ENAP) - Criação de curso de capacitação em gestão de TI; (5) TCU/SEFTI - publicação de dados sobre governança de TI. Conteúdo mínimo para Termo de Referência/Projeto Básico, definições para Pregão de TI. Além de divulgação de informações consolidados em sumários executivos e informativos de TI nos órgãos e entidades da APF

Os resultados do trabalho do TCU geraram mudanças significativas nas áreas de TI da APF. Foi notória a evolução da governança em TI e da gestão dos contratos de TI.

\subsubsection{Realidade do MCTI}

No âmbito do MCTI, através da Coordenação-Geral de Gestão de TI (CGTI), foi criado o Núcleo de Contratação de TI, onde analistas foram alocados no órgão e os servidores com Gratificação Temporária do SISP (GSISP) fiscalizavam seus contratos.

Porém, apesar de todo o avanço, foi detectado que não era o suficiente, pois mesmo com os mecanismos existentes, a eficiência preconizada ainda não estava sendo obtida. Além disso, diversas outras habilidades não estavam sob o domínio dos servidores lotados na CGTI, tais como: gerenciamento de risco; comunicação; negociação com os envolvidos; garantia da qualidade; controle de mudanças; e monitoramento e controle de cronograma. As atividades de gestão e controle de contratos de TI, basicamente, voltaram-se à conformidade financeiro-contratual dos projetos, não se atendo mais ao gerenciamento dos requisitos, planejamento, verificação e monitoramento dos riscos e demais disciplinas de projeto, tão pouco com a validação de código-fonte produzido, a adequação das decisões arquiteturais tomadas e outras questões de desenvolvimento de SI.

Dessa forma, a CGTI percebeu uma grande oportunidade de realizar uma reestruturação na forma de realizar suas competências. Aproveitou a Portaria 101 de criação do Escritório de Projetos ${ }^{2}$ e a chegada dos novos servidores com

\footnotetext{
${ }^{1}$ http://www.governoeletronico.gov.br/sispconteudo/nucleo-de-contratacoes-de-ti/modelode-contratacoes-normativos-e-documentos-dereferencia/instrucao-normativa-mp-slti-no04/

${ }^{2}$ http://pesquisa.in.gov.br/imprensa/jsp/visualiza/ index.jsp?jornal $=1 \&$ pagina $=26 \&$ data $=12 / 07 / 2012$
}

experiência em processos, gerenciamento de projetos e desenvolvimento de software para realizar uma verdadeira mudança de cultura na condução dos projetos de TI do MCTI.

A reestruturação envolvia uma nova abordagem: centrar na capacitação do servidor em gerenciamento de projetos. Não apenas provendo conhecimento, mas criando e padronizando todos os processos e disponibilizando ferramentas para a condução de suas atividades.

\section{PROJETO DE REESTRUTURAÇÃO}

Para operacionalizar os objetivos deste trabalho, foi utilizada uma metodologia que compreende o estudo, o desenvolvimento prático e teórico da proposta de reestruturação, que inclui as seguintes atividades: explorar a partir de um ponto de vista teórico a estratégia de implantanção do Escritório de Projetos de Tecnologia da Informação (EPTI) no contexto do MCTI; desenvolver a estratégia proposta para implantação EPTI, uma vez que isto é realizado a partir da compreensão teórica dos estudos anteriores e de outras teorias relacionadas; desenvolver e melhorar os processos internos relacionados ao desenvolvimento de SI; customizar e implantar ferramentas de suporte; capacitar os servidores nas disciplinas relacionadas; implantar os processos e ferramentas para validação da nova abordagem; e avaliar qualitativamente os primeiros resultados com a implantação da nova estrutrura de TI.

Nessa direção, a reformulação da estrutura de TI da CODS foi realizada com base em três pilares básicos, conforme apresentado na Figura 1.

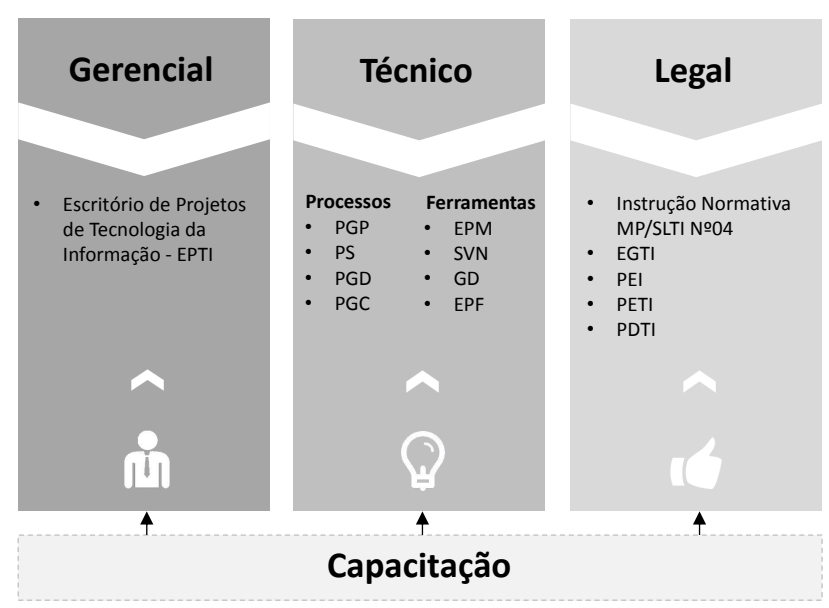

Figura 1: Projeto de Reestruturação da TI

1. Gerencial: estruturação de um Escritório de Projetos de Tecnologia da Informação (EPTI);

2. Técnico: implementação de um processo de Gerenciamento de Projetos baseado no guia Project Management Book of Knowledge (PMBok) [11]; desenvolvimento e implantação de um processo de desenvolvimento de software baseada no Capability Maturity Model Integration (CMMI) [12]; desenvolvimento do processo de gerenciamento de demandas e gerenciamento da configuração; e promoção de capacitações e workshops. 
3. Legal: desenvolvimento de Processo de Gestão de Demandas aderente à IN 04 do MP/SLTI, com foco na orquestração de serviços prestados por empresas terceirizadas.

Cada pilar contou com a produção de normas e procedimentos internos da CGTI, aliada à uma política de capacitação, acompanhamento e mentoring em projetos.

\subsection{Pilar Gerencial}

Com vista ao aperfeiçoamento da gestão de processos de TI na APF, o EPTI foi estruturado para exercer um papel subjacente no processo de crescimento do MCTI, que é o de contribuir para a eficiência nos resultados dos seus projetos estratégicos através de uma série responsabilidades.

O posicionamento organizacional, os papéis, as responsabilidades e o funcionamento do EPTI são regidos por seu Modelo Operacional (MO-EPTI). O organograma da Figura 1 ilustra o posicionamento do EPTI na estrutura informal da CGTI.

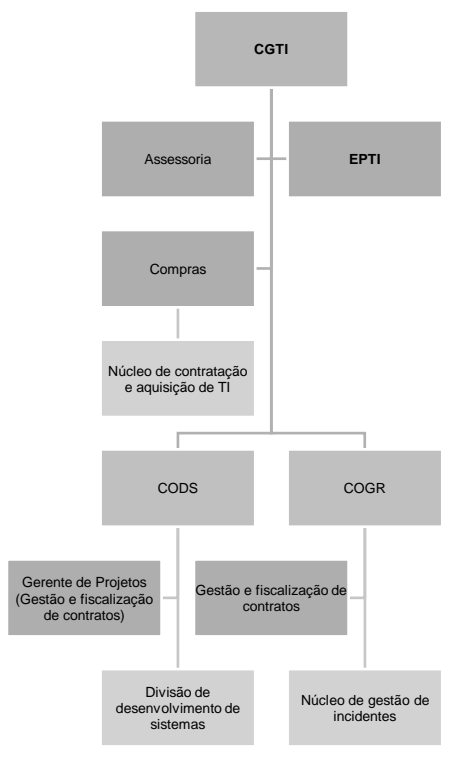

Figura 2: Organograma da CGTI

Atualmente, o EPTI abrange o portfólio de projetos relacionados à CODS, implementando um escritório de projetos do tipo departamental (tático), assumindo a função de suporte, i.e. o EPTI é responsável por desenvolver e implantar processos e melhores práticas para o gerenciamento de projetos com abrangência no âmbito da CODS/CGTI/MCTI, fornecer treinamento, ferramentas, software de gestão de projetos e demandas. Nesse tipo de implantação, o EPTI não assume responsabilidade pelos resultados dos projetos.

Levando em consideração as competências atribuídas ao EPTI, para concretização de seu propósito, implementamse cinco grupos independentes de funções: (1) desenvolvimento de competências, processos e metodologias; (2) monitoramento e controle de indicadores de desempenho; (2) gerenciamento de múltiplos projetos; (4) gerenciamento estratégico; (5) e aprendizagem organizacional.

Visando subsidiar a implantação do EPTI, foi elaborada uma estratégia de implantação do escritório de projetos na
CODS, que também forneceu uma estimativa de horas requeridas. Essa iniciativa forneceu uma base sólida para a definição do modelo de atuação do Escritório de Projetos, em seu primeiro ano, aderente às necessidades diagnosticadas durante este processo, bem como apoiou a definição de estratégias para a construção do MO-EPTI. Esta estratégia de implementação do EPTI foi composta de 3 fases:

1. Diagnóstico, definição e institucionalização do processo padrão de Gerenciamento de Projetos (PGP-MCTI);

2. Definição e planejamento do EPTI;

3. Elaboração, implantação e acompanhamento do EPTI.

Essas fases foram compostas por diversas atividades, como: diagnóstico, planejamento, treinamentos, definição e validação dos processos de Gerênciamento de Projetos (GP) e de um PMO.

Com base nessas atribuições e funções, o EPTI tem por finalidade atuar na resolução de diversos problemas, como e.g padronizar a execução dos projetos; consolidar as informações dos projetos, controlar a utilização dos recursos de pessoal e financeiro, melhorar o controle de informações dos contratos, auxiliar a fiscalização e gestão dos contratos, entre outros. Também pode-se vislusbrar oportunidades a serem exploradas, como e.g. a criação de uma cultura de GP, alcançar um nível de maturidade em GP e alcançar eficência no planejamento e condução do projetos.

Dentre as diversas atividades que foram executadas pelo EPTI, pode-se destacar:

- Diagnóstico do nível de maturidade em GP e organizacional através da metodologia Organizational Project Management Maturity Model (OPM3) [1];

- Criação de processos padronizados para o GP e demandas;

- Elaboração de normativo para proposição e GP;

- Capacitação dos fornecedores em GP;

- Fornecimento de informações consolidadas dos projetos;

- Criação de ferramentas de apoio à tomada de decisão contemplando relatórios, consultas e painéis (Dashboards) de apoio à fiscalização de contratos, controle de ordens de serviço e projetos;

- Promoção de visibilidade perante à CGTI das ações do CODS;

- Capacitação dos servidores em ferramentas e técnicas de GP, engenharia de software e gerência da configuração, além de workshops, mentoring e coaching;

- Controle efetivo dos riscos dos projetos.

\subsection{Pilar Técnico}

Concernente ao pilar técnico foi necessário desenvolver e melhorar processos internos do MCTI, bem como customizar ferramentas de apoio, promover capacitações e apoio à tomada de decisão, conforme descrito na sequência. 


\subsubsection{Melhoria de processos}

Os processos internos do MCTI foram concebidos com o objetivo de suportar, em um nível satisfatório de maturidade, o gerenciamento e execução de projetos e demandas de TI no âmbito do MCTI através da descrição de um conjunto de diretrizes, processos, critérios, artefatos, regras e padrões, imprescindíveis para a execução de projetos com qualidade, produtividade e segurança. Estes processos encontram-se em evolução constante visando incorporar as lições aprendidas.

Dentre todos os componentes e atividades que foram desenvolvidas para todos os processos que foram definidos, pode-se destacar: elaboração de um documento descritivo contendo todas as fases do ciclo de vida, as disciplinas, grupos de processo, papéis e responsabilidades e todos os artefatos que contemplam o processo; mapeamento das atividades que foram definidas no PS-MCTI para as Áreas de Processo (AP) do Capability Maturity Model Integration (CMMI) [12]; elaboração de Guias Operacionais (GO), Instruções de Trabalho e Qualidade (ITQ) e Listas de Verificação (LV). Todos os documentos anteriormente citados estão disponíveis no repositório do EPTI para acesso interno. Adicionalmente, todos os processos também foram implementados na ferramenta Eclipse Process Framework Project $(\mathrm{EPF})^{3}$, que fornece um framework extensível e ferramentas para a engenharia de processo de software, i.e. um método e processo de criação e gerenciamento de bibliotecas, configuração e a publicação de um processo.

\section{Processo de gerenciamento de projetos}

O PGP-MCTI foi customizado a partir das melhores práticas de mercado, brainstormings internos e outras fontes como PMBOK [11]. Ele está dividido em cinco categorias que formam um macro-processo, aqui chamadas grupos de processo para manter a conformidade com o PMBOK [11]. Cada grupo de processo descreve uma série de atividades de gerenciamento de projetos, onde a saída de um processo pode ser utilizada como entrada em outro processo. Os grupos de processos não são fases dos projetos, pois podem ser executados de forma sobreposta ao longo do projeto. As interações entre os grupos de processos estão ilustrados na Figura 3.

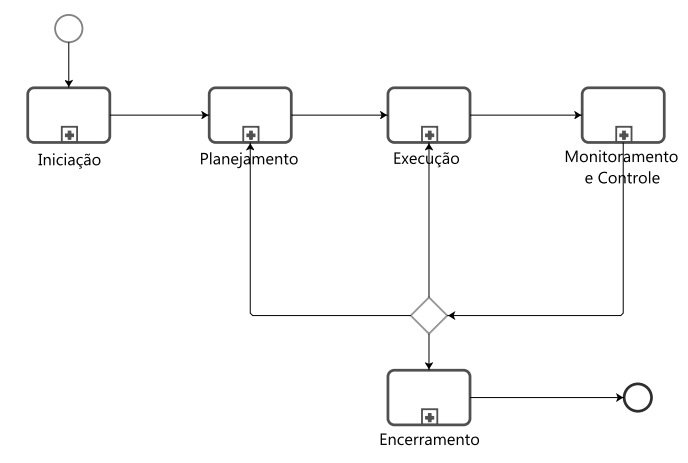

\section{Figura 3: Grupos de processos do PGP-MCTI}

Cada grupo de processo possui em seu fluxo de trabalho um conjunto de atividades que englobam artefatos de entrada e saída, responsávís por sua execução, critérios de

\footnotetext{
${ }^{3}$ http://www.eclipse.org/epf/
}

entrada e saída e descrição. Para exemplificar, o grupo de processo "planejamento" está ilustrado na Figura 4

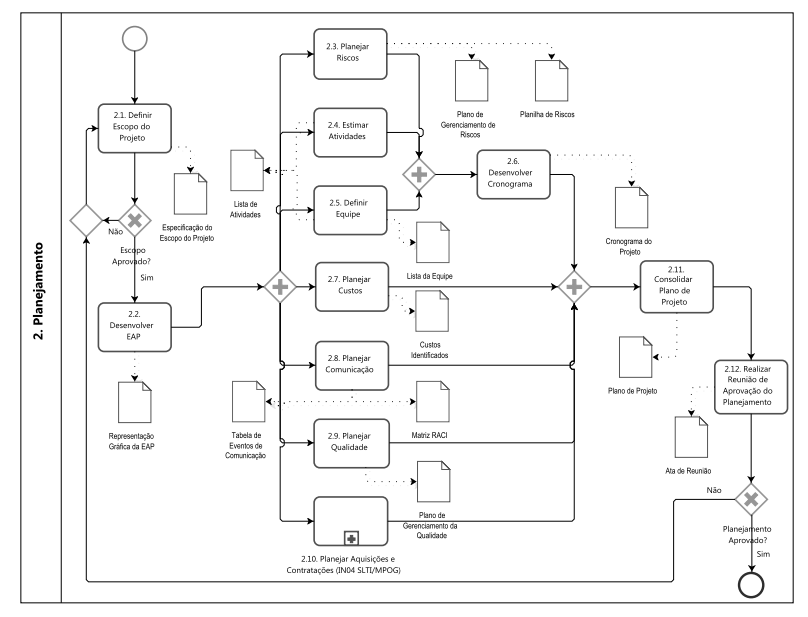

Figura 4: Processo de planejamento do PGP-MCTI

No âmbito da CODS, em toda demanda de "projeto" é atribuído um gerente de projeto, servidor público desse departamento, responsável por executar as atividades que foram definidas no processo com o objetivo final de potencializar as chances de sucesso do projeto.

\section{Processo de software}

As versões anteriores do PS-MCTI (versão 1.0 e 2.0), no momento e contexto em que foram concebidas, estavam voltadas para os processos de gestão e fiscalização contratuais, conforme descrito na IN 04 . No entanto, ocorriam diversos problemas relacionados a qualidade do produto e processo, que em sua grande parte eram resultados obtidos a partir do baixo nível de maturidade. Motivado por estes problemas e também baseado nas recomendações dos órgãos de controle (Seção 2.2), foi necessário redefinir o PS-MCTI com base em modelos, normas e padrões de qualidade de software internacionalmente aceitos pela indústria e comunidade acadêmica.

Nesse contexto, foi elaborada a versão 3.0 do PS-MCTI visando elevar de forma gradativa o nível de maturidade no desenvolvimento de software, com base nas melhores práticas de mercado, brainstormings internos e os seguintes modelos: PMBOK [11]; Rational Unified Process (RUP) [7]; CMMI [12]; Test Maturity Model Integration (TMMi) [13]; entre outros. Optou-se pelo CMMI por ser o modelo mais conhecido internacionalmente que vem sendo adotado por diversas empresas de software que estão buscando um diferencial de qualidade. O CMMI inspirou outros modelos, como o MPS.BR ${ }^{4}$ e também mantem a conformidade com as normas ISO/IEC 15504 [5] e ISO 12207 [4]

Devido ao contexto da APF para a customização do PSMCTI foi utilizada representação contínua do CMMI, pois foi necessário tornar alguns processos mais maduros. Desta forma, foram implementadas doze AP's do CMMI, satisfazendo todas as práticas específicas, garantindo que os fluxos de atividades definidas no processo estejam aderentes aos objetivos e práticas exigidas no CMMI.

As AP's do CMMI[12] que foram implementadas são: Planejamento de Projeto (PP); Monitoramento e Controle de

$\overline{{ }^{4} \text { http://www.softex.br/mpsbr/ }}$ 
Projeto (MCP); Gestão de Configuração (GC); Medição e Análise (MA); Garantia da Qualidade de Processo e Produto (PPQA); Gestão de Riscos (RSKM); Gestão de Requisitos (REQM; Desenvolvimento de Requisitos (RD); Solução Técnica (ST); Integração de Produto (IP); Validação (VAL); e Verificação (VER). O relacionamento entre as fases, disciplinas, grupos de processo e suas respectivas categorias está ilustrado na Figura 5.

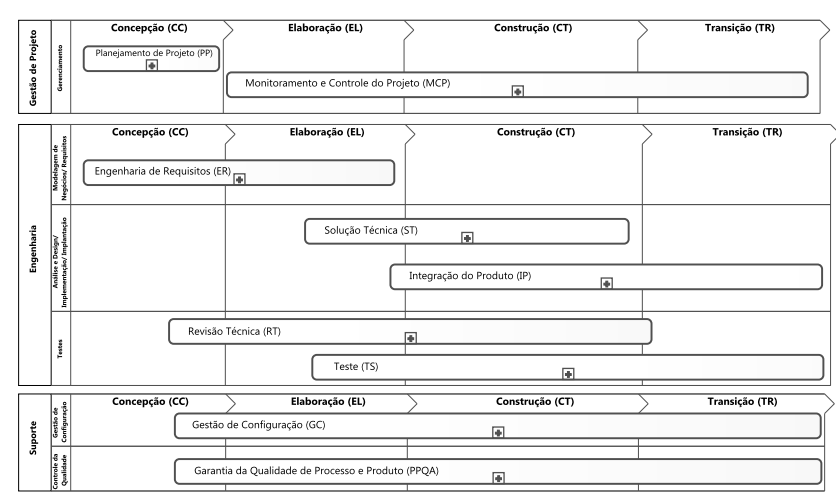

Figura 5: Relacionamento entre os componentes do PS-MCTI

O ciclo de vida do PS-MCTI é baseado no desenvolvimento iterativo incremental, possibilitando que o modelo seja utilizado em projetos de software de tamanhos variados, suportando projetos onde não seja possível definir o problema e construir o software em um único passo, entre outras vantagens relacionadas à integração, implementação, requisitos, riscos e arquitetura.

\section{Processo de gestão de demandas}

Alinhado ao pilar relacionado à legalidade da gestão e fiscalização dos contratos de TI, o PGD-MCTI, pela sua flexibilidade, garante um efetivo controle sobre as demandas de TI e abrange os seguintes objetivos principais:

- Atender as necessidades do MCTI, transformando as demandas internas em projetos e manutenções, melhorando consequentemente a qualidade do atendimento do serviço interno/externo.

- Garantir um nível de governança das solicitações, como visibilidade, controle e transparência, assim como a justificativa alinhada com o planejamento estratégico da organização;

- Proporcionar à CGTI maior visibilidade sobre todas as demandas das áreas do MCTI, atendendo tais demandas com os recursos humanos e financeiros disponíveis;

- Fornecer um valor mensurável de projetos, de forma rápida, reduzindo o risco de falhas e cancelamentos de projetos e manutenções;

- Garantir o alinhamento à legislação pertinente, principalmente como uma ferramenta de apoio à gestão e fiscalização de contratos de serviços.

Dentre os principais benefícios pode-se destacar: a redução da probabilidade de se investir em iniciativas que não estejam alinhadas aos objetivos estratégicos; visibilidade das demandas operacionais e estratégicas; garantia de alinhamento e conformidade ao PGP-MCTI e PS-MCTI; melhoria no gerenciamento através de indicadores de performance, métricas e relatórios; customização do ciclo das demandas para atender a dinâmica de negócio do MCTI; e apoio na tomada de decisão e a priorização do portfólio de projetos.

O PGD-MCTI compreende três subprocessos de gestão, cada um composto por uma série de atividades para a gestão de demanda e suas interações: Projeto, Manutenção e Ordem de Serviço (OS).

\section{Processo de gerenciamento da configuração}

Conforme contextualizado na Seção 2.2, diversas atividades da CGTI são executadas por empresas terceirizadas, incluindo o desenvolvimento de aplicações de TI. No PS-MCTI estão contempladas atividades de gerenciamento da configuração, mas nos casos em que ocorrem o desenvolvimento de aplicações por empresas terceirizadas, a execução das atividades de GC previstas no PS-MCTI são também realizadas por tais empresas em seus respectivos ambientes de trabalho e o MCTI não conseguia ter visibilidade e controle destas versões. A CGTI deve gerenciar em sua infraestrutura a configuração de todos esses Itens de Configuração (IC) desenvolvidos por teceiros, com uma visão real das versões em sua própria infraestrutura, tanto de documentos como de código-fonte, que foram entregues e que estão nos ambientes de desenvolvimento, testes, homologação e produção.

Desta forma, independente do processo de GC que foi implementado no PS-MCTI, é necessário que a CGTI tenha um processo interno de GC. Consequentemente, foi desenvolvido o PGC-MCTI que tem por finalidade padronizar os procedimentos necessários para as atividades de GC. Este processo define o método para geração e controle de Itens de Configuração (IC) e Configurações Base (CB) para todos as aplicações de TI no âmbito da CODS.

Em linhas gerais, o PGC-MCTI descreve os conceitos básicos para identificação da configuração de documentos e código-fonte, define todo o processo de controle de versão, fluxo de versionamento de documentos e código-fonte, desenvolvimento em paralelo, estruração de armazenamento e políticas de acesso, e fluxo de publicação de código-fonte em todos ambientes (desenvolvimento, testes, homologação e produção).

\subsubsection{Customização de ferramentas}

Embora a implementação do EPTI e a definição de diversos processos contribuiram com a melhoria da qualidade no desenvolvimento de projetos e demandas na CODS, foi necessário customizar algumas ferramentas para apoiar em diversas atividades no escopo deste trabalho.

\section{Ferramentas para gerenciamento de demandas e pro- jetos}

Um grande desafio é automatizar procedimentos, metodologias ou processos com foco na otimização e medição do processo para tomada de decisões utilizando indicadores chave sobre todo o fluxo de trabalho.

Desta forma, visando atender as necessidades de GP da CODS, foi implantada a plataforma Microsoft Enterprise Project Management(EPM) ${ }^{5}$ integrado com o Microsoft Sha-

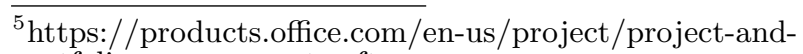
portfolio-management-software 
repoint ${ }^{6}$. O EPM é um sistema de informação para gerenciamento de projetos corporativos, através do qual são aplicadas técnicas de gestão elencadas pelo $\mathrm{PMI}^{7}$ no PMBOK [11]. Entretanto, somente ter a ferramenta, sem a devida utilização das técnicas de gestão aplicadas de maneira adequada, o sistema EPM torna-se ineficiente, sem um objetivo geral e sem agregar a devida funcionalidade para a organização.

Diante do exposto, em busca de inovação e aperfeiçoamento, o EPM foi customizado com base no PGD-MCTI e PGP-MCTI com o objetivo de atender às necessidades do MCTI. Nesta direção, foram automatizados os respectivos fluxos de trabalho dos processos no ambiente EPM.

Visando apoiar a tomada de decisão, uma das maiores preocupações da CGTI era estar municiada de informações inerentes aos projetos de sua responsabilidade de forma consolidada que fossem confiáveis, íntegras e disponível de maneira rápida e acessível. Nesse sentido, o EPTI elaborou painéis de consultas gerenciais que demonstram, de forma gráfica, a real situação dos projetos e das demandas existentes na CGTI.

Ferramentas para o gerenciamento da configuração

Para suportar o PGC-MCTI, a ferramenta escolhida pela CODS foi o sistema de controle de versão Subversion ${ }^{8}$ (SVN), e como cliente o TortoiseSvn ${ }^{9}$. O SVN é uma ferramenta de controle de versão que permite o desenvolvimento colaborativo a partir de um repositório único, tendo como as principais vantagens o rastreamento das alterações realizadas durante o desenvolvimento de software ou de um documento de texto qualquer.

Dentre as funções do SVN pode-se destacar: controle do histórico - possibilidade de analisar o histórico do desenvolvimento, permitindo analisar as alterações com detalhes, desde a primeira versão até a última, podendo resgatar versões mais antigas e estáveis ou até desfazer o trabalho; trabalho em equipe - permite que diversas pessoas trabalhem sobre o mesmo conjunto de documentos ao mesmo tempo, minimizando o desgaste provocado por problemas com conflitos de edições; marcação e resgate de versões estáveis permite marcar onde é que o documento estava com uma versão estável, podendo ser facilmente resgatado no futuro; ramificação de projeto - possibilita a divisão do projeto em várias linhas de desenvolvimento, que podem ser trabalhadas paralelamente, sem que uma interfira na outra.

Como o SVN é uma ferramenta de fácil integração com outros softwares, ele foi integrado ao ambiente EPM/Sharepoint visando obter um ambiente único e consolidado para gerenciamento das demandas e projetos.

\subsubsection{Capacitações}

Para conseguir um maior impacto na reestruturação da TI foi necessário uma mudança cultural na CGTI, i.e. para que os servidores tenham maior eficiência na execução dos processos e atividades que foram estruturados, foi necessário investir no desenvolvimento de determinadas habilidades. Nesta direção, foram realizadas capacitações e workshops em diversas disciplinas e ferramentas, tais como:

\footnotetext{
$\overline{{ }^{6} \text { http://products.office.com/en- }}$

us/sharepoint/collaboration

${ }^{7}$ http://www.pmi.org/

${ }^{8}$ https://subversion.apache.org/

${ }^{9}$ http://tortoisesvn.net/
}

- Capacitações em Gerenciamento de projetos: (1) Gerenciamento de projetos PMI: uma abordagem prática; (2) Microsoft Project 2013 - treinamento oficial; (3) Gestão de projetos e portfólios com Microsoft EPM.

- Workshop em processos: (1) Processo de gerenciamento de projetos; (2) Processo de gerenciamento de demandas; (3) Processo de software; (4) Processo de gerenciamento da configuração.

- Capacitação em Gerência da Configuração: Treinamento básico de TortoiseSVN e Controle de Versão.

\subsection{Pilar Legal}

Todo o projeto de reestruturação, além de buscar atingir um maior nível de maturidade da organização e aumentar a chance de sucesso nos projetos de TI, também foi baseado em diversas exigências legais, como recomendações de órgãos de controle interno e externo, IN 04, entre outras.

Visando cumprir determinações de acórdãos do TCU e relatórios de auditoria da Controladoria-Geral da União (CGU), foi estabelecido o PS-MCTI versão 3.0 considerando as normas ISO/IEC 12.207, ISO/IEC 15.504 e também considerando uma ampla discussão, validação e aprovação entre as àreas competentes. As mesmas recomendações também resultaram na implantação de uma estrutura formal de GP, à semelhança das orientações do processo PO10.2 contidas no COBIT [6] e no PMBOK, conforme descrito no PGP-MCTI.

Em outro relatório de auditoria da CGU, em relação ao PS-MCTI, também foi recomendado avaliar a conveniência e oportunidade de: revisar periodicamente o processo; capacitar os recursos humanos na modelagem e melhoria de processos; melhorar os processos de comunicação entre os envolvidos; desenvolver um processo de gerenciamento da configuração; e garantir a qualidade e melhoria do nível de maturidade do processo. Tais recomendações foram contempladas na definição PS-MCTI versão 3.0 e também no plano de capacitações e workshops que foram organizados na CGTI.

Visando atender a Estratégia Geral de Tecnologia da Informação (EGTI) do SISP, foi criado o EPTI no âmbito da CGTI/MCTI. Apesar do EPTI exercer diversas funções, possuir uma série de competências e gerar diversos benefícios, o EPTI também atende ao disposto na EGTI.

A IN 04, que fornece um guia prático para a contratação de soluções de TI, no que se refere à gestão e fiscalização dos contratos, está sendo atendida através do PGD-MCTI, que além de uniformizar o processo de gestão das demandas, fornece saídas que atendem ao disposto na instrução normativa.

\section{RESULTADOS E DISCUSSÃO}

O projeto de reestruturação exigiu um grande esforço técnico nos trabalhos de definição de processos, instalação, customização de ferramentas e capacitação dos servidores em diversas áreas, conforme descrito na Seção 3.

No início da implantação do EPTI, foi realizado um diagnóstico baseado na metodologia OPM3 [1] com o objetivo de avaliar o grau de maturidade gerencial, onde através de um questionário envolvendo as melhores práticas de GP, constatou-se que em apenas alguns aspectos, a CGTI possuia um nível de maturidade sob demanda (ad-hoc - até $33 \%$ dos processos implementados), sendo que alguns processos, 
como o caso de qualidade e riscos, não existia nenhuma prática implementada.

Também foi realizada uma análise da versão 2.0 do PSMCTI e de práticas de gerenciamento da configuração no MCTI, onde foram constatadas diversas questões que impactavam no insucesso dos projetos, conforme descrito na Seção 3.2.1. Desta forma, foram elaborados pareceres técnicos que balizaram as oportunidades de melhoria e reestruturação da área de TI.

Após tais identificações iniciais, partiu-se para a fase de projeto e desenvolvimento da reestruturação da área de TI. Em seguida, iniciou-se a fase de implantação, onde os projetos existentes no órgão foram direcionados para as seguintes ações: (1) projetos na fase de execução - foram analisados e cada um foi direcionado para o estágio correto do processo; (2) projetos em fase de planejamento - a orientação foi para realizarem as atividades de iniciação.

Uma vez ajustados, os projetos tiveram seu curso normal no processo e o EPTI realizou as verificações de execução e, de forma não invasiva, realizou orientações e direcionamentos junto aos gerentes de projetos. As demandas que surgem, estão sendo incluídas no portfólio de projetos EPTI e seguindo todos processos que foram definidos.

Apesar da recente implantação desta nova reestruturação, pode-se destacar alguns resultados preliminares:

- Gerenciamento do portfólio de projetos de forma padronizada e consolidada através do PGP-MCTI e suporte da ferramenta EPM;

- Suporte à tomada de decisão através de painéis de informações consolidadas na ferramenta EPM;

- O EPTI, através de auditorias, verificações e acompanhamento nos projetos, atua como guardião das práticas de gerenciamento de processos e projetos de TI na organização;

- O EPTI auxilia na formação e capacitação de servidores em gerenciamento de projetos, processos e ferramentas;

- A ênfase no planejamento proporciona maior assertividade nos prazos e custos do projeto, cujos indicadores podem ser obtidos através da consolidação e controle dos projetos na ferramenta EPM;

- Aumento da qualidade dos produtos, melhoria da comunicação, otimização no uso dos recursos; estabelecimento de vocabulário comum e adequação às especificidades do MCTI, através de processos bem definidos e maduros;

- Consolidação de conhecimentos e experiências adquiridos através do registro de lições aprendidas;

- Caracterização do nível de maturidade em Gerência de Projetos e Desenvolvimento de Software através da implantação de processos aderentes às normas e padrões internacionais.

\section{CONCLUSÃO}

Nesse trabalho foi apresentado um relato de experiência que demonstra o projeto de reestruturação da área de TI no MCTI, o qual através da reformulação e implantação de processos, ferramentas de suporte e mudança da cultura organizaçional, foi possível aumentar a maturidade organizacional proporcionado aumento no índice de sucesso dos projetos de TI e agregação de valor ao negócio do MCTI.

Por fim, observa-se como oportunidade futura a experimentação dos processos que foram definidos em mais projetos de TI do MCTI, visando identificar eventuais gargalos, oportunidades de melhoria e também a conveniência de introduzir práticas ágeis de GP e desenvolvimento de software. Também, faz-se necessário a evolução da ferramenta EPM no sentido de automatizar outras atividades dos processos, bem como proporcionar formas alternativas de visualização das informações.

\section{Referências}

[1] Organizational Project Management Maturity Model (Opm3) Overview. Project Management Institute, 1st edition, 2003. ISBN 1930699042.

[2] F. M. M. da Silva, V. Sun, J. P. de Albuquerque, and E. P. V. Prado. O Funil de Inovação como Modelo para Priorizar e Executar Projetos de Tecnologia da Informação. In Simpósio Brasileiro de Sistemas de Informação - SBSI, 2014.

[3] G. D. Garson and A. Pavlichev. Digital government: principles and best practices, 2004.

[4] ISO/IEC. ISO/IEC 12207. Information technology software life-cycle processes, 1995.

[5] ISO/IEC. ISO/IEC 15504. Iinformation technology process assessment. Technical report, 2003-2006.

[6] IT Governance Institute, editor. CobiT 4.1: Framework, Control Objectives, Management Guidelines, Maturity Models. IT Governance Institute, Rolling Meadows, 2007.

[7] P. Kruchten. The Rational Unified Process: An Introduction. Addison-Wesley, 2003. ISBN 0201707101.

[8] K. C. Laudon and J. P. Laudon. Management Information Systems: Managing the Digital Firm. Prentice Hall PTR, Upper Saddle River, NJ, USA, 10th edition, 2006. ISBN 0130330663.

[9] D. Lima, R. Silva, A. Garcia, S. Rodrigues, M. Chaves, R. Santos, and J. Souza. Sistema para Transição de Governos no Brasil. In Simpósio Brasileiro de Sistemas de Informação - SBSI, 2013.

[10] A. P. Martins, M. R. Martins, M. M. M. Pereira, and V. A. Martins. Implantação e consolidação de escritório de gerenciamento de projetos: um estudo de caso. Production, 15:404 - 415, 12 2005. ISSN 0103-6513.

[11] PMI. A Guide to the Project Management Body of Knowledge PMBOK Guide, 5th Edition. Project Management Institute, 2012.

[12] S. E. I. Team. CMMI for Development, v1.3 (CMU/SEI-2010-TR-033). Software Engineering Institute, Carnegie Mellon University, 2010.

[13] E. v. Veenendaal and B. Wells. Test Maturity Model Integration TMMi. Uitgeverij Tutein Nolthenius, The Netherlands, 2012. ISBN 9490986100, 9789490986100. 